INPLASY

PROTOCOL

To cite: Zhao et al. Thyroid diseases are associated with coronavirus disease 2019

(COVID-19) infection. Inplasy protocol 202190079. doi:

10.37766/inplasy2021.9.0079

Received: 23 September 2021

Published: 23 September 2021

Corresponding author:

Yaru Mou

mouyaru1983@163.com

Author Affiliation:

The First Affiliated Hospital of

Shandong First Medical

University \& Shandong

Provincial Qianfoshan

Hospital.

Support: ZR2019PH025.

Review Stage at time of this submission: Formal screening of search results against eligibility criteria.

Conflicts of interest:

None declared.

\section{Thyroid diseases are associated with coronavirus disease 2019 (COVID-19) infection}

Zhao, J1; Tian, Y2; Wang, H³ Yao, J4; Song, W5; Mou, Y6.

Review question / Objective: The objective is to discusses the impact of thyroid disease on new coronary pneumonia through a meta-analysis, and provides new treatment ideas for the later treatment and recovery of new coronary pneumonia.

Information sources: Pubmed, Embase, Cochrane library, Sinomed, CNKI and Wanfang, were conducted for studies of thyroid diseases in patients with COVID infection. The literature search for this meta-analysis was restricted to published results. Databases were searched from the earliest data to 4 November 2020 with the search terms: ("coronavirus disease 2019" OR "COVID-19") AND ("thyroid diseases" OR "hyperthyroidism" OR "hypothyroidism" OR "thyroid function").

INPLASY registration number: This protocol was registered with the International Platform of Registered Systematic Review and Meta-Analysis Protocols (INPLASY) on 23 September 2021 and was last updated on 23 September 2021 (registration number INPLASY202190079).

\section{INTRODUCTION}

Review question / Objective: The objective is to discusses the impact of thyroid disease on new coronary pneumonia through a meta-analysis, and provides new treatment ideas for the later treatment and recovery of new coronary pneumonia.
Condition being studied: Coronavirus is an enveloped single-stranded positivestranded RNA virus of the Coronavirus family. The new 2019 novel Coronavirus (2019-nCoV) is currently the seventh known coronavirus that can infect humans. Coronavirus (CoV) usually causes mild to moderate infection of the respiratory tract, 
accompanied by the typical symptoms of a cold or flu infection, namely a runny nose, cough, sore throat, headache, sub-fever or fever. In addition to the above-mentioned typical clinical conditions, coronaviruses can also cause serious diseases of the lower respiratory tract, which are more common in people with heart or lung or malignant tumors, immunosuppression, infants and the elderly. The clinical manifestations of new coronary pneumonia are very similar to SARS-CoV. Critically ill patients develop Acute Respiratory Distress Syndrome (ARDS) and require Intensive Care Unit (ICU) admission and oxygen therapy. In addition, the study found that patients infected with 2019nCoV also have a large amount of Interleukin-1B(IL1B), Interferon $v$ (IFNY), Human Neutrophils Protein10 (IP10) and mono-cyte chemotactic protein-1(MCP1), which may cause activated T-helper-1 (Th1) cell responses. In addition, patients who require ICU admission have higher granulocyte colony stimulating factor (GCSF), IP10, MCP1, Macrophage Inflammatory Protein 1 Alpha (MIP1A) and Tumor Necrosis Factor Alpha (TNFa) concentrations than patients who do not require ICU admission, which indicates that the cytokine concentration is related to the severity of the disease. Evidence to date shows that having chronic diseases increases the risk of serious illnesses from COVID-19. In addition, there are data on the vulnerability of patients taking immunosuppressants to treat inflammatory chronic diseases. They are more susceptible to COVID-19 infection and tend to develop into more serious infections. Studies have found that the overall mortality rate of hospitalized patients with elevated levels of procalcitonin (PCT) among COVID-19 infected patients is higher than that of patients with normal PCT levels. The study found that abnormal thyroid function is also related to the severity of respiratory diseases. Among 61 severe acute respiratory syndrome (SARS) survivors, a study was conducted three months after recovery, and four (6.6\%) were diagnosed It was primary hypothyroidism, and thyroid lesions were found in the autopsy of patients who died of SARS.

\section{METHODS}

Participant or population: Patients with COVID-19.

Intervention: The severity of COVID-19 infection.

Comparator: No severe patients.

Study designs to be included: the cohort study or case series.

Eligibility criteria: Eligible studies met the following criteria: (1) published in English or Chinese language; (2) study assessed the association between COVID infection and thyroid diseases; (3) study was designed as the cohort study or case series; (4) study reported at least one result including but not limited to the number of cases, prevalence, et al.

Information sources: Pubmed, Embase, Cochrane library, Sinomed, CNKI and Wanfang, were conducted for studies of thyroid diseases in patients with COVID infection. The literature search for this meta-analysis was restricted to published results. Databases were searched from the earliest data to 4 November 2020 with the search terms: ("coronavirus disease 2019" OR "COVID-19") AND ("thyroid diseases" OR "hyperthyroidism" OR "hypothyroidism" OR "thyroid function").

Main outcome(s): Number of patients with thyroid disease

Quality assessment / Risk of bias analysis: Studies were independently screened by two reviewers and disagreements were resolved by consensus.

Strategy of data synthesis: The main outcome was the number of patients with thyroid diseases in patients with COVID-19 infection. Other outcome, the number of patients with thyroid diseases in patients with severe disease of COVID-19 infection was also collected. Fixed-model performed 
by computing odds ratio (OR) and $95 \% \mathrm{Cl}$ for dichotomous variables. The I2was calculated as an index of heterogeneity between studies. The analyses were performed by Review Manager 5.3 (Cochrane Collaboration, United Kingdom,http://www.cochrane.org).

Subgroup analysis: Subgroup analysis was further conducted by types of thyroid diseases.

Sensitivity analysis: The 12 was calculated as an index of heterogeneity between studies.

Country(ies) involved: China.

Keywords: thyroid diseases, hypothyroidism, coronavirus disease 2019, COVID-19, meta-analysis.

Contributions of each author:

Author 1 - Junyu Zhao.

Author 2 - Yutian Tian.

Author 3 - Haipeng Wang.

Author 4 - Jinming Yao.

Author 5 - Wang Song.

Author 6 - Yaru Mou. 